\title{
Predictors of postoperative complications after robot-assisted radical cystectomy with extracorporeal urinary diversion
}

This article was published in the following Dove Press journal: Cancer Management and Research

\author{
Chung Un Lee* \\ Minyong Kang* \\ Tae Jin Kim \\ Jun Phil $\mathrm{Na}$ \\ Hyun Hwan Sung \\ Hwang Gyun Jeon \\ Seong II Seo \\ Seong Soo Jeon \\ Hyun Moo Lee \\ Byong Chang Jeong
}

Department of Urology, Samsung Medical Center, Sungkyunkwan University School of Medicine, Seoul, Republic of Korea

*These authors contributed equally to this work
Correspondence: Byong Chang Jeong Department of Urology, Samsung Medical Center, Sungkyunkwan University School of Medicine, 8I irwon-ro, gangnam-gu, Seoul 0635I, Republic of Korea

Tel +82234100737

Fax +82234106992

Email bc2.jung@samsung.com
Purpose: Robot-assisted radical cystectomy (RARC) is known to have less postoperative morbidity and complications than open radical cystectomy. However, various complications not yet have been reported after RARC. In this study, we aimed to identify the predictors of complications following RARC.

Patients and methods: From August 2008 to November 2017, we retrospectively reviewed 126 patients who underwent RARC with extracorporeal urinary diversion. Overall perioperative complications were examined, and factors that may affect complications were analyzed using a logistic regression model. Complications were classified according to the ClavienDindo system.

Results: Overall postoperative complications occurred in $78(61.9 \%)$ of 126 patients. Whereas the rate of minor complications was $58.0 \%$ (grade $\mathrm{I}=15.9 \%(\mathrm{n}=20)$, grade $\mathrm{II}=42.1 \%(\mathrm{n}=53)$ ), the rate of major complications was very low (grade IIIa=1.6\% $(n=2)$, grade IIIb $=2.4 \%(n=3)$ ). No fatal complications more than grade IV were developed. Notably, transfusions (27.0\%), urinary tract infection (15.9\%), anastomosis site leakage (14.3\%), and ileus $(10.3 \%)$ were the most common complications after RARC. In the multivariate regression model, previous intravesical instillation (odds ration $[\mathrm{OR}]=3.374)$, preoperative hemoglobin $(\mathrm{OR}=0.751)$, and estimated blood loss $(\mathrm{EBL})(\mathrm{OR}=3.949)$ were identified as the predictors of postoperative complications.

Conclusion: In sum, our data showed the rates of major complications were comparable after RARC with extracorporeal urinary diversion compared as reported in previous studies and lower major than minor complications following RARC. Moreover, we identified the independent predictors of postoperative complications, such as preoperative hemoglobin, intravesical instillation, and EBL.

Keywords: bladder cancer, complications, cystectomy, predictor, robotic surgery

\section{Introduction}

Bladder cancer is one of the most common genitourinary cancers in the world and is associated with a high morbidity and mortality rate. Because of its aggressiveness, many studies focus on the optimal treatment of bladder cancer. ${ }^{1}$ According to the European Association of Urology guidelines, radical cystectomy is recommended not only for patients with muscle-invasive bladder cancer, but also for patients with non-muscle invasive bladder cancer who are at highest risk of progression and patients with BCG (bacillus calmette-guérin)-refractory bladder cancer., ${ }^{2,3}$ However, radical cystectomy is one of the most complex urological surgical procedures, with higher complication rates. 
Minimally invasive robot-assisted radical cystectomy (RARC) is the most advanced surgical approach to reduce the incidence of complications. ${ }^{4}$ Postoperative morbidity and mortality after RARC are lower than those in open radical cystectomy (ORC), while the oncologic outcomes are comparable. ${ }^{5-8}$

While the proportion of overall postoperative complications after RARC has decreased compared with complication rates of ORC (57-74\%) due to advances in surgical methods and techniques, it still remains high at 35-63\%. Similarly, RARC is associated with fewer major complications more than grade III or more than ORC (about 22\%), but the rate of major complications is still high $10 \%{ }^{9-11}$

To reduce the incidence of complications following surgery, it is important to predict and prevent factors associated with the occurrence of complications. High American Society of Anesthesiologists (ASA) score, estimated blood loss (EBL), Charlson comorbidity index (CCI), and obesity are well-known predictors of perioperative complications in patients undergoing ORC. ${ }^{12,13}$ However, there is still a lack of research in postoperative complications and associated risk factors were still limited in patients underwent RARC.

Here, we evaluated postoperative complications following RARC with extracorporeal urinary diversion, and identified predictive factors for these complications following RARC with extracorporeal urinary diversion.

\section{Material and methods}

We retrospectively reviewed 126 consecutive patients who underwent RARC with extracorporeal urinary diversion from August 2008 to November 2017. Age at the time of surgery, sex, body mass index (BMI), ASA score, preoperative hemoglobin, history of previous abdominal surgery, history of intravesical instillation, history of neoadjuvant chemotherapy, pathologic stage, operative time, EBL, and type of urinary diversion were included as demographic data and perioperative data. This study was approved by the International Review Board of Samsung Medical Center (approval number: 2018-05-146) with the Declaration of Helsinki. For this type of study, formal consent was not required and data were anonymous.

Complications were classified according to the modified Clavien-Dindo system. ${ }^{14}$ Major complications were defined as Clavien-Dindo grade III or greater, while minor complications were defined as Clavien-Dindo grade II or less. Multiple complications were defined as the presence of more than one complication in the same patient.
The following descriptive statistics were used for continuous data: mean, standard deviation, and $95 \%$ confidence interval (CI) or median and interquartile range (IQR). Predictive factors for complications after RARC were determined using a multivariate logistic regression model and calculating the odds ratio (OR) and 95\% CI. Multivariate analyses were performed with only significant factors as judged by univariate analysis.

Statistical analyses were performed using SAS version 9.4 (SAS Institute, Cary, NC, USA) and R 3.4.3 (Vienna, Austria; http://www.R-project.org/). Values of $p<0.05$ were considered statistically significant.

\section{Results}

\section{Patient characteristics}

Baseline demographics of patients are presented in Table 1. The mean age of patients with RARC was 61.7 \pm 10.7 . RARC was performed primarily in males $(91.3 \%$, $\mathrm{n}=115$ ). Mean preoperative hemoglobin was $13.4 \pm 1.8$, and patients who had previously undergone abdominal surgery comprised $15.9 \%(\mathrm{n}=20)$. Among patients with RARC, $20.6 \%(n=26)$ underwent intravesical instillation (except for three patients who received epirubicin intravesical instillation), and all patients underwent BCG intravesical instillation, while $15.1 \%(\mathrm{n}=19)$ of patients underwent neoadjuvant chemotherapy. T3 pathology was the most common (37.3\%, $\mathrm{n}=47)$, and LN (lymph node) metastasis was confirmed in $27.8 \%(n=35)$ of patients (Table 1).

\section{Perioperative data}

Perioperative data are shown in Table 2. Median overall operation time was 500 minutes (IQR, 435-585), median EBL was $400 \mathrm{~mL}$ (IQR, 300-600), median hospital stay was 23 days (IQR, 17-32), and median follow-up was 22.4 months (IQR, 8.0-43.0). The diversion type of RARC was preferred to ileal conduit $(68.3 \%, \mathrm{n}=86)$ compared to neobladder formation $(31.7 \%, \mathrm{n}=40)$. In patients with ileal neobladder, longer operative times (527 minutes [IQR, 465-655] vs 445 minutes [IQR, 372-526]) and longer hospital stays (25 days [IQR, 20-33] vs 17 days [IQR, 16-21]) were recorded than patients with ileal conduit. However, EBL did not significantly differ between ileal neobladder and ileal conduit (450 minutes [IQR, 300-600] vs 400 minutes [IQR, $300-600])$. Transfusions were performed in 34 patients (27\%), most of which were performed postoperatively (30 patients, $23.8 \%$ ), with intraoperative transfusions performed in 8 patients $(6.3 \%)$ (Table 2$)$. 
Table I Patient demographics

\begin{tabular}{|c|c|}
\hline Variable & Value \\
\hline Age, year & $61.7 \pm 10.7$ \\
\hline \multicolumn{2}{|l|}{ Sex, n (\%) } \\
\hline Male & $115(91.3)$ \\
\hline Female & II (8.7) \\
\hline \multicolumn{2}{|l|}{ BMI, n (\%) } \\
\hline$<18.5$ & $5(4.0)$ \\
\hline $18.5-22.9$ & $50(39.7)$ \\
\hline $23.0-24.9$ & $26(20.6)$ \\
\hline$\geq 25.0$ & $45(35.7)$ \\
\hline Preoperative hemoglobin & $13.4 \pm 1.8$ \\
\hline \multicolumn{2}{|l|}{$\mathrm{CCl}, \mathrm{n}(\%)$} \\
\hline 3 or lower & $44(34.9)$ \\
\hline 4 & $41(32.5)$ \\
\hline 5 & $24(19.0)$ \\
\hline 6 or higher & $17(13.5)$ \\
\hline \multicolumn{2}{|l|}{ ASA score, $n$ (\%) } \\
\hline 1 & $37(29.4)$ \\
\hline 2 & $84(66.7)$ \\
\hline 3 & $5(4.0)$ \\
\hline \multicolumn{2}{|l|}{ Previous abdominal surgery, $\mathrm{n}(\%)$} \\
\hline Yes & $20(15.9)$ \\
\hline No & $106(84.1)$ \\
\hline \multicolumn{2}{|l|}{ Intravesical instillation, n (\%) } \\
\hline Yes* & $26(20.6)$ \\
\hline No & $100(79.4)$ \\
\hline \multicolumn{2}{|l|}{ Neoadjuvant chemotherapy, n (\%) } \\
\hline Yes & $19(15.1)$ \\
\hline No & $107(84.9)$ \\
\hline \multicolumn{2}{|l|}{ Pathologic stage, n (\%) } \\
\hline T0 & $7(5.6)$ \\
\hline $\mathrm{Ta}$ & $\mathrm{I}(0.8)$ \\
\hline Tcis & $10(7.9)$ \\
\hline $\mathrm{TI}$ & $23(18.3)$ \\
\hline $\mathrm{T} 2$ & $26(20.6)$ \\
\hline T3 & $47(37.3)$ \\
\hline $\mathrm{T} 4$ & $12(9.5)$ \\
\hline LN metastasis, $\mathrm{n}(\%)$ & $35(27.8)$ \\
\hline
\end{tabular}

Notes: *Except for 3 (epirubicin intravesical instillation), all patients underwent BCG intravesical instillation.

Abbreviations: BMI, body mass index; $\mathrm{CCl}$, Charlson comorbidity index; ASA, American Society of Anesthesiologists; LN, lymph node; BCG, bacillus calmette-guérin.

\section{Complications}

Complications occurred in 78 patients $(61.9 \%)$. Total 95 minor complications occurred in 73 patients (57.9\%), 5 major complications occurred in 5 patients
Table 2 Perioperative data

\begin{tabular}{|l|l|}
\hline Variable & Value \\
\hline Median overall operation time, min & $500(435-585)$ \\
lleal conduit & $445(372-526)$ \\
Neobladder (studer) & $527(465-655)$ \\
\hline Median EBL, $\mathrm{mL}$ & $400(300-600)$ \\
Ileal conduit & $400(300-500)$ \\
Neobladder (studer) & $450(300-600)$ \\
\hline LN removal, $\mathrm{n}$ (\%) & \\
Yes & $120(95.2)$ \\
Mean LN removal & $20.1 \pm 8.9$ \\
No & $6(4.8)$ \\
\hline Type of urinary diversion, $\mathrm{n}(\%)$ & \\
Ileal conduit & $40(31.7)$ \\
Neobladder (studer) & $86(68.3)$ \\
\hline Transfusion, $\mathrm{n}$ (\%) & $34(27.0)$ \\
Intraoperative & $8(6.3)$ \\
Postoperative & $30(23.8)$ \\
\hline Surgical margin positive, $\mathrm{n}(\%)$ & \\
Yes & $9(7.1)$ \\
Ureter & $6(4.8)$ \\
Perivesical tissue & $2(1.6)$ \\
Vas deferens & $1(0.8)$ \\
No & $117(92.9)$ \\
\hline Median hospital stays, day & $23(17-32)$ \\
Ileal conduit & $17(16-2 \mathrm{I})$ \\
Neobladder (studer) & $25(20-33)$ \\
\hline Time to flatus, day & $3.6 \pm 3.2$ \\
\hline Median follow up, month & \\
Neal conduit & $9.1-43.0)$ \\
\hline
\end{tabular}

Abbreviations: EBL, estimated blood loss; LN, lymph node.

(4.0\%), and multiple complications occurred in 22 patients $(17.5 \%)$.

Grade I complication occurred in 27 patients (21.4\%), grade II complication occurred in 68 patients $(54.0 \%)$, grade IIIa complication occurred in 2 patients (1.6\%), and grade IIIb complication occurred in 3 patients $(2.4 \%)$. Complication of grade IV or higher did not occur (Table 3).

Detailed complications following RARC are listed in Table 4. The most frequent complications were transfusion $(27.0 \%, \mathrm{n}=34)$, febrile urinary tract infection $(15.9 \%, \mathrm{n}=20)$, anastomosis site leakage $(14.3 \%, \mathrm{n}=18)$, and ileus $(10.3 \%$, $\mathrm{n}=13$ ). A classification of complications and specific complications according to diversion type and pathologic results are listed in Tables S1 and 2. In addition, a classification of 
Table 3 Clavien-Dindo classifications of complications following RARC

\begin{tabular}{|l|l|}
\hline & No. (\%) \\
\hline Patients with complications, n (\%) & $78(61.9)$ \\
Patients with minor complications, $\mathrm{n}(\%)$ & $73(57.9)$ \\
Patients with major complications, $\mathrm{n}(\%)$ & $5(4.0)$ \\
Patients with multiple complications, $\mathrm{n}(\%)$ & $22(17.5)$ \\
\hline Total number of complications & 100 \\
Grade I complications, $\mathrm{n}(\%)$ & $27(27.0)$ \\
Grade II complications, $\mathrm{n}(\%)$ & $68(68.0)$ \\
Grade III a or greater complications, $\mathrm{n}(\%)$ & $5(5.0)$ \\
\hline Highest complications grade, $\mathrm{n}(\%)$ & \\
No complications & $48(38.1)$ \\
Grade I & $20(15.9)$ \\
Grade II & $53(42.1)$ \\
Grade IIIa & $2(1.6)$ \\
Grade IIIb & $3(2.4)$ \\
Grade IV & $0(0)$ \\
Grade V & $0(0)$ \\
\hline
\end{tabular}

Abbreviations: RARC, robot-assisted radical cystectomy; EBL, estimated blood loss; LN, lymph node.

Table 4 Detailed complications following RARC

\begin{tabular}{|l|l|}
\hline Complications & No. (\%) \\
\hline Transfusion & $34(27.0)$ \\
UTI (febrile) & $20(15.9)$ \\
Anastomosis site leakage & $18(14.3)$ \\
Ileus & $13(10.3)$ \\
\hline Wound problem & $5(4.0)$ \\
Grade I & $2(1.6)$ \\
Grade IIIa & $2(1.6)$ \\
Grade IIIb & $1(0.8)$ \\
\hline Lymphocele & $2(1.6)$ \\
Bowel injury & $2(I .6)$ \\
\hline Operation site fluid collection & $2(I .6)$ \\
Grade I & $1(0.8)$ \\
Grade IIIa & $1(0.8)$ \\
\hline Scrotal edema & $2(I .6)$ \\
Anastomosis site stricture & $1(0.8)$ \\
Pulmonary thromboembolism & $1(0.8)$ \\
\hline
\end{tabular}

Abbreviations: RARC, robot-assisted radical cystectomy; UTI, urinary tract infection.

complications and specific complications according to intravesical instillation are also listed in Table S3.

\section{Predictors of complications}

In univariate analysis, intravesical instillation $(\mathrm{OR}=3.168$, 95\% $\mathrm{CI}=1.10-9.07, p=0.0318)$ and $\mathrm{EBL}(\mathrm{OR}=3.159,95 \%$
$\mathrm{CI}=1.452-6.876, p=0.0037)$ were significant predictors associated with complications following RARC. In multivariate analysis, intravesical instillation $(\mathrm{OR}=3.374,95 \%$ $\mathrm{CI}=1.116-10.197, p=0.0312)$, EBL $(\mathrm{OR}=3.949,95 \%$ $\mathrm{CI}=1.682-9.27, p=0.0016$ ), and preoperative hemoglobin $(\mathrm{OR}=0.751,95 \% \mathrm{CI}=0.588-0.96, p=0.022)$ were significant predictors associated with complications following RARC (Table 5.).

\section{Discussion}

In this study, we investigated complications following RARC and factors affecting the occurrence of complications after RARC. We found that preoperative hemoglobin, EBL, and intravesical instillation were significant predictors of complications following RARC.

Previous studies have shown that overall complication rates after RARC vary from center to center. According to Al-Daghmin et al in RARC with mainly extracorporeal conduit diversion, the overall complication rate is $77 \%$, the low-grade complication rate is $58 \%$, and the high-grade complication rate is $19 \% .{ }^{15}$ According to Yuh et al in RARC with mainly extracorporeal continent diversion, the overall complication rate is $82 \%$, the low-grade complication rate is $45 \%$, and the high-grade complication rate is $37 \% .{ }^{16}$ According to Nazmy et al in RARC with mainly extracorporeal continent diversion, the overall complication rate is $77 \%$, the low-grade complication rate is $45 \%$, and the high-grade complication rate is $32 \% .{ }^{17}$ In contrast, we found that the incidence of overall complications after RARC was lower $(61.9 \%)$ in our study. While rates of low-grade (Clavien-Dindo grade 1 or 2) complications after RARC (57.9\%) were similar, rates of high-grade complications (Clavien-Dindo grade 3 or 4) were much lower than those in previous reports $(4 \%)$.

Many previous studies have explored predictors of complications following RARC. According to Nazmy et al, ASA, preoperative hematocrit, and diversion type contribute to any grade of complications after RARC. ${ }^{17}$ Yuh et al identified age, ASA, preoperative hematocrit, operation time, EBL, and diversion type as predictors of complications after RARC. ${ }^{18}$ However, according to Abe et al there were no significant differences in overall complications according to diversion type, ${ }^{19}$ while according to Tan et al preoperative anemia is not associated with increased preoperative complications in patients treated with RARC. ${ }^{20}$ Notably, we identified intravesical instillation $(\mathrm{OR}=3.374)$ as a novel unfavorable predictive factor for complications after RARC, in addition to preoperative 
Table 5 Logistic regression model to identify predictive factors for postoperative complications following RARC

\begin{tabular}{|c|c|c|c|c|}
\hline & \multicolumn{2}{|c|}{ Univariable analysis } & \multicolumn{2}{|c|}{ Multivariable analysis } \\
\hline & OR $(95 \% \mathrm{Cl})$ & $p$ & OR $(95 \% \mathrm{CI})$ & $p$ \\
\hline Age & $0.996(0.963-1.031)$ & 0.8333 & & \\
\hline $\begin{array}{l}\text { Sex } \\
\qquad \text { Male } \\
\text { Female }\end{array}$ & $1.714(0.432-6.803)$ & $\begin{array}{l}\text { Reference } \\
0.4438\end{array}$ & & \\
\hline $\begin{array}{l}\text { BMI } \\
\quad<25 \\
\geq 25\end{array}$ & $0.763(0.362-1.609)$ & $\begin{array}{l}\text { Reference } \\
0.4775\end{array}$ & & \\
\hline Preoperative hemoglobin & $0.816(0.661-1.008)$ & 0.0594 & $0.751(0.588-0.96)$ & 0.022 \\
\hline $\begin{array}{l}\text { ASA score } \\
\qquad \begin{array}{l}1 \\
2 \\
3\end{array}\end{array}$ & $\begin{array}{l}0.807(0.322-2.018) \\
1.652(0.144-18.944)\end{array}$ & $\begin{array}{l}0.7174 \\
\text { Reference } \\
\mathrm{I} \\
\mathrm{I}\end{array}$ & & \\
\hline $\begin{array}{l}\text { Abdominal surgery history } \\
\text { No } \\
\text { Yes }\end{array}$ & I.53। (0.545-4.3) & $\begin{array}{l}\text { Reference } \\
0.4187\end{array}$ & & \\
\hline $\begin{array}{l}\text { Urinary diversion type } \\
\text { Conduit } \\
\text { Neobladder }\end{array}$ & $1.778(0.828-3.821)$ & $\begin{array}{l}\text { Reference } \\
0.1402\end{array}$ & & \\
\hline $\begin{array}{l}\text { Intravesical instillation } \\
\text { No } \\
\text { Yes }\end{array}$ & $3.168(1.106-9.078)$ & $\begin{array}{l}\text { Reference } \\
0.0318\end{array}$ & $3.374(1.116-10.197)$ & $\begin{array}{l}\text { Reference } \\
0.0312\end{array}$ \\
\hline $\begin{array}{l}\text { Neoadjuvant chemotherapy } \\
\text { No } \\
\text { Yes }\end{array}$ & $0.821(0.305-2.212)$ & $\begin{array}{l}\text { Reference } \\
0.6964\end{array}$ & & \\
\hline $\begin{array}{l}\text { EBL } \\
\text { LN removal } \\
\text { Operation time } \\
\text { Operation year }\end{array}$ & $\begin{array}{l}3.159(1.452-6.876) \\
0.97 \mid(0.935-1.009) \\
1.001(0.999-1.004) \\
0.92(0.812-1.042)\end{array}$ & $\begin{array}{l}0.0037 \\
0.1305 \\
0.3373 \\
0.1913\end{array}$ & $3.949(1.682-9.27)$ & 0.0016 \\
\hline
\end{tabular}

Abbreviations: RARC, robot-assisted radical cystectomy; OR, odds ratio; Cl, confidence interval; BMI, body mass index; ASA, American Society of Anesthesiologists; EBL, estimated blood loss; LN, lymph node.

hemoglobin $(\mathrm{OR}=0.751)$ and $\mathrm{EBL}(\mathrm{OR}=3.949)$. Because intravesical instillation was a significant factor for complications following RARC, more careful procedures are needed when operating on patients with intravesical instillation history. Additionally, preoperative hemoglobin control through anemia correction or nutritional support and careful bleeding control during surgery are necessary to reduce complications after RARC.

We hypothesized that there are unknown effects of intravesical instillation resulting in complications, including anatomical modifications such as mucosal changes in the bladder and deterioration of immunity after performing transurethral resection of the bladder several times before intravesical instillation or intravesical instillation. In fact, bladder contracture or necrosis has been reported with local side effects after BCG intravesical instillation, ${ }^{21}$ and a previous study found that BCG intravesical instillation causes modulation of peripheral immune mechanisms in addition to local immune activation. ${ }^{22}$

There are some limitations of this study. First, the study was retrospective and analyzed a relatively small quantity of data. Second, we conducted the study in a single tertiary center and cannot rule out selection bias. Third, we included only RARC with extracorporeal urinary diversion, so it is 
difficult to generalize our results to RARC as a whole. Fourth, our analysis did not reflect the improvement of the surgeon's RARC technique over time. More accurate results would be obtained by analyzing the learning curve of the surgeon. Finally, our results may reflect minor complications rather than major complications, because few major complications occurred. Additionally, in this study we do not adjust enhanced recovery after surgery (ERAS) protocol to all patients because the ERAS protocol introduced in urologic surgery relatively recently.

Briefly mention about ERAS protocol, it is a multimodal perioperative care pathway designed to achieve early recovery for patients undergoing major surgery. ${ }^{23}$ ERAS protocol was applied first at colorectal surgery to decrease complications after surgery and introduced in urologic surgery especially in radical cystectomy relatively recently. ${ }^{24}$ There is a meta-analysis of the effect of ERAS protocol on perioperative outcomes after radical cystectomy that shows reduction in length of the index hospitalization, decrease in the rate of low-grade complications, and improvement of the time-tobowel function, so the benefit of applying the ERAS protocol to radical cystectomy is expected to be substantial. ${ }^{25} \mathrm{We}$ expect the further reduction of complications by applying ERAS protocol to RARC, especially in ileus.

Nevertheless, our study has strength in relatively large amount of RARC cases from single institution. And it is significant that our study reemphasizes exisiting predictors of complications following RARC and presents new predictors, such as history of intravesical instillation.

\section{Conclusion}

We detected that the rates of major complications were comparable after RARC with extracorporeal urinary diversion compared as reported in previous studies and lower major than minor complications following RARC in our study. The predictors of postoperative complications following RARC were intravesical instillation, preoperative hemoglobin, and EBL during surgery. Our results offer valuable information that may help clinicians to reduce complications following RARC.

\section{Acknowledgments}

This study was funded by a research grant from the National Research Foundation (NRF) of Korea, funded by the Ministry of Science and Information \& Communication Technology (ICT) (2017R1A2B4010568). This research was also supported by a grant of the Korea Health Technology R\&D Project through the Korea Health
Industry Development Institute (KHIDI), funded by the Ministry of Health \& Welfare, Republic of Korea (HI17C0025). The abstract of this paper was presented at the 38th Congress of the Société Internationale d'Urologie (SIU) as a poster presentation with interim findings. The poster's abstract was published in "Poster Abstracts" in World Journal of Urology (https://doi.org/10.1007/ s00345-018-2492-3).

\section{Disclosure}

The authors report no conflicts of interest in this work.

\section{References}

1. Klotz L, Brausi MA. World urologic oncology federation bladder cancer prevention program: a global initiative. Urol Oncol. 2015;33 (1):25-29. doi:10.1016/j.urolonc.2014.07.017

2. Alfred Witjes J, Lebret T, Comperat EM, et al. Updated 2016 EAU guidelines on muscle-invasive and metastatic bladder cancer. Eur Urol. 2017;71(3):462-475. doi:10.1016/j.eururo.2016.06.020

3. Babjuk M, Bohle A, Burger M, et al. EAU guidelines on non-muscleinvasive urothelial carcinoma of the bladder: update 2016. Eur Urol. 2017;71(3):447-461. doi:10.1016/j.eururo.2016.05.041

4. Menon M, Hemal AK, Tewari A, et al. Nerve-sparing robot-assisted radical cystoprostatectomy and urinary diversion. BJU Int. 2003;92 (3):232-236

5. Sung HH, Ahn JS, Seo SI, et al. A comparison of early complications between open and robot-assisted radical cystectomy. $J$ Endourol. 2012;26(6):670-675. doi:10.1089/end.2011.0372

6. Bak DJ, Lee YJ, Woo MJ, et al. Complications and oncologic outcomes following robot-assisted radical cystectomy: what is the real benefit? Investig Clin Urol. 2016;57(4):260-267. doi:10.4111/icu.2016.57.4.260

7. Ram D, Rajappa SK, Rawal S, Singh A, Singh PB, Dewan AK. Is robot-assisted radical cystectomy superior to standard open radical cystectomy? An Indian perspective. J Minim Access Surg. 2018. doi:10.4103/jmas.JMAS_150_17

8. Kader AK, Richards KA, Krane LS, Pettus JA, Smith JJ, Hemal AK. Robot-assisted laparoscopic vs open radical cystectomy: comparison of complications and perioperative oncological outcomes in 200 patients. BJU Int. 2013;112(4):E290-294. doi:10.1111/bju.12167

9. Khan MS, Elhage O, Challacombe B, Rimington P, Murphy D, Dasgupta P. Analysis of early complications of robotic-assisted radical cystectomy using a standardized reporting system. Urology. 2011;77(2):357-362. doi:10.1016/j.urology.2010.04.063

10. Hayn MH, Hellenthal NJ, Hussain A, Stegemann AP, Guru KA. Defining morbidity of robot-assisted radical cystectomy using a standardized reporting methodology. Eur Urol. 2011;59 (2):213-218. doi:10.1016/j.eururo.2010.10.044

11. Kauffman EC, Ng CK, Lee MM, et al. Critical analysis of complications after robotic-assisted radical cystectomy with identification of preoperative and operative risk factors. BJU Int. 2010;105 (4):520-527. doi:10.1111/j.1464-410X.2009.08843.x

12. Bostrom PJ, Kossi J, Laato M, Nurmi M. Risk factors for mortality and morbidity related to radical cystectomy. BJU Int. 2009;103 (2):191-196. doi:10.1111/j.1464-410X.2008.07889.x

13. Hirobe M, Tanaka T, Shindo T, et al. Complications within 90 days after radical cystectomy for bladder cancer: results of a multicenter prospective study in Japan. Int J Clin Oncol. 2018;23:734-741. doi:10.1007/s10147-018-1245-z

14. Dindo D, Demartines N, Clavien P-A. Classification of surgical complications. Ann Surg. 2004;240(2):205-213. 
15. Al-Daghmin A, Aboumohamed A, Din R, et al. Readmission after robot-assisted radical cystectomy: outcomes and predictors at 90-day follow-up. Urology. 2014;83(2):350-356. doi:10.1016/j. urology.2013.09.056

16. Yuh B, Torrey RR, Ruel NH, et al. Intermediate-term oncologic outcomes of robot-assisted radical cystectomy for urothelial carcinoma. J Endourol. 2014;28(8):939-945. doi:10.1089/end.2014.0073

17. Nazmy M, Yuh B, Kawachi M, et al. Early and late complications of robot-assisted radical cystectomy: a standardized analysis by urinary diversion type. J Urol. 2014;191(3):681-687. doi:10.1016/j. juro.2013.10.022

18. Yuh BE, Nazmy M, Ruel NH, et al. Standardized analysis of frequency and severity of complications after robot-assisted radical cystectomy. Eur Urol. 2012;62(5):806-813. doi:10.1016/j.eururo.2012.06.007

19. Abe T, Takada N, Shinohara N, et al. Comparison of 90-day complications between ileal conduit and neobladder reconstruction after radical cystectomy: a retrospective multi-institutional study in Japan. Int J Urol. 2014;21(6):554-559. doi:10.1111/iju.12357

20. Tan WS, Lamb BW, Khetrapal P, et al. Blood transfusion requirement and not preoperative anemia are associated with perioperative complications following intracorporeal robot-assisted radical cystectomy. J Endourol. 2017;31(2):141-148. doi:10.1089/end.2016.0730
21. Kronig M, Jilg C, Burger D, et al. BCG induced necrosis of the entire bladder urothelium. Urol Case Rep. 2015;3(5):161-163. doi:10.1016/j. eucr.2015.06.002

22. ElsÄSser-Beile U, Gutzeit O, Bauer S, Katzenwadel A, SchultzeSeemann W, Wetterauer U. Systemic and local immunomodulatory effects of intravesical BCG therapy in patients with superficial urinary bladder carcinomas. J Urol. 2000;163(1):296-299.

23. Kehlet H. Multimodal approach to control postoperative pathophysiology and rehabilitation. Br J Anaesth. 1997;78(5):606-617.

24. Geltzeiler CB, Rotramel A, Wilson C, Deng L, Whiteford MH, Frankhouse J. Prospective study of colorectal enhanced recovery after surgery in a community hospital. JAMA Surg. 2014;149 (9):955-961. doi:10.1001/jamasurg.2014.675

25. Tyson MD, Chang SS. Enhanced recovery pathways versus standard care after cystectomy: a meta-analysis of the effect on perioperative outcomes. Eur Urol. 2016;70(6):995-1003. doi:10.1016/j. eururo.2016.05.031 


\section{Supplementary materials}

Table SI Clavien-Dindo classifications of postoperative complications and specific complications following RARC according to type of urinary diversion

\begin{tabular}{|c|c|c|}
\hline & Ileal conduit $(\mathbf{N}=40)$ & Ileal neobladder $(\mathbf{N}=86)$ \\
\hline Patients with complications, n (\%) & $21(52.5)$ & $57(66.3)$ \\
\hline Patients with minor complications, $\mathrm{n}$ (\%) & $19(47.5)$ & $54(62.8)$ \\
\hline Patients with major complications, $\mathrm{n}(\%)$ & $2(5.0)$ & $3(3.0)$ \\
\hline Patients with multiple complications, n (\%) & $7(17.5)$ & $15(17.4)$ \\
\hline Total number of complications & 29 & 71 \\
\hline Minor complications, n (\%) & $27(93.1)$ & $68(95.8)$ \\
\hline Major complications, n (\%) & $2(6.9)$ & $3(4.2)$ \\
\hline \multicolumn{3}{|l|}{ Highest complications grade, $n$ (\%) } \\
\hline No complications & $19(47.5)$ & $29(33.7)$ \\
\hline Grade I & $4(10.0)$ & $16(1.6$ \\
\hline Grade II & $15(37.5)$ & $38(44.2)$ \\
\hline Grade IIIa & $\mathrm{I}(2.5)$ & $\mathrm{I}(\mathrm{I} .2)$ \\
\hline Grade IIIb & $\mathrm{I}(2.5)$ & $2(2.3)$ \\
\hline \multicolumn{3}{|l|}{ Specific complications, n (\%) } \\
\hline Transfusion & $15(37.5)$ & $19(22.1)$ \\
\hline UTI (febrile) & $\mathrm{I}(2.5)$ & $19(22.1)$ \\
\hline Anastomosis site leakage & $\mathrm{I}(2.5)$ & $17(19.8)$ \\
\hline lleus & $4(10.0)$ & $9(10.5)$ \\
\hline
\end{tabular}

Abbreviations: RARC, robot-assisted radical cystectomy; UTI, urinary tract infection.

Table S2 Clavien-Dindo classifications of postoperative complications and specific complications following RARC according to pathologic results

\begin{tabular}{|c|c|c|}
\hline & T0, Tcis, Ta, TI, T2 (N=67) & $\mathrm{T3}, \mathrm{T} 4(\mathrm{~N}=59)$ \\
\hline Patients with complications, $\mathrm{n}(\%)$ & $44(65.7)$ & $34(57.6)$ \\
\hline Patients with minor complications, $\mathrm{n}(\%)$ & $39(58.2)$ & $34(57.6)$ \\
\hline Patients with major complications, $\mathrm{n}(\%)$ & $5(7.5)$ & $0(0.0)$ \\
\hline Patients with multiple complications, n (\%) & $14(20.9)$ & $8(13.6)$ \\
\hline Total number of complications & 58 & 42 \\
\hline Minor complications, n (\%) & $53(91.4)$ & $42(100.0)$ \\
\hline Major complications, n (\%) & $5(8.6)$ & $0(0)$. \\
\hline \multicolumn{3}{|l|}{ Highest complications grade, $\mathrm{n}(\%)$} \\
\hline No complications & $23(34.3)$ & $25(42.4)$ \\
\hline Grade I & $9(13.4)$ & II (18.6) \\
\hline Grade II & $30(44.8)$ & $23(39.0)$ \\
\hline Grade IIIa & $2(3.0)$ & $0(0.0)$ \\
\hline Grade IIIb & $3(4.5)$ & $0(0.0)$ \\
\hline \multicolumn{3}{|l|}{ Specific complications, $\mathrm{n}(\%)$} \\
\hline Transfusion & $18(26.9)$ & $16(27.1)$ \\
\hline UTI (febrile) & $12(17.9)$ & $8(13.6)$ \\
\hline Anastomosis site leakage & $10(14.9)$ & $8(13.6)$ \\
\hline Ileus & $8(11.9)$ & $5(8.5)$ \\
\hline
\end{tabular}

Abbreviations: RARC, robot-assisted radical cystectomy; UTI, urinary tract infection. 
Table S3 Clavien-Dindo classifications of postoperative complications and specific complications following RARC according to intravesical instillation

\begin{tabular}{|c|c|c|}
\hline & Intravesical instillation $(\mathbf{N}=\mathbf{2 6})$ & No intravesical instillation $(\mathrm{N}=100)$ \\
\hline Patients with complications, n (\%) & $21(80.8)$ & $57(57.0)$ \\
\hline Patients with minor complications, $n$ (\%) & $27(103.8)$ & $68(54.0)$ \\
\hline Patients with major complications, $\mathrm{n}$ (\%) & I (3.8) & $4(4.0)$ \\
\hline Patients with multiple complications, n (\%) & $6(23.1)$ & $16(16.0)$ \\
\hline Total number of complications & 28 & 72 \\
\hline Minor complications & $27(96.4)$ & $68(94.4)$ \\
\hline Major complications & I (3.6) & $4(5.6)$ \\
\hline \multicolumn{3}{|l|}{ Highest complications grade, $n$ (\%) } \\
\hline No complications & $5(19.2)$ & $43(43.0)$ \\
\hline Grade I & $4(15.4)$ & $16(16.0)$ \\
\hline Grade II & $16(61.5)$ & $37(37.0)$ \\
\hline Grade IIIa & I (3.8) & $\mathrm{I}(\mathrm{I} .0)$ \\
\hline Grade IIIb & $0(0)$ & $3(3.0)$ \\
\hline \multicolumn{3}{|l|}{ Specific complications, n (\%) } \\
\hline Transfusion & $10(38.5)$ & $24(24.0)$ \\
\hline UTI (febrile) & $7(26.9)$ & $13(13.0$ \\
\hline Anastomosis site leakage & $3(11.5)$ & $15(15.0)$ \\
\hline lleus & $3(11.5)$ & $10(10.0)$ \\
\hline
\end{tabular}

Abbreviations: RARC, robot-assisted radical cystectomy; UTI, urinary tract infection.

\section{Publish your work in this journal}

Cancer Management and Research is an international, peer-reviewed open access journal focusing on cancer research and the optimal use of preventative and integrated treatment interventions to achieve improved outcomes, enhanced survival and quality of life for the cancer patient.
The manuscript management system is completely online and includes a very quick and fair peer-review system, which is all easy to use. Visit http://www.dovepress.com/testimonials.php to read real quotes from published authors. 